\title{
Decoupled and simultaneous three-dimensional imaging and optical manipulation through a single objective: supplementary material
}

\author{
Arran Curran ${ }^{1},{ }^{*}$, Simon Tuohy ${ }^{2}$, Dirk G. A. L. Aarts ${ }^{1}$, Martin J. Booth $^{2}$, \\ TONY Wilson ${ }^{2}$, AND ROEL P. A. DULLENS ${ }^{1}$ \\ ${ }_{1}^{1}$ Department of Chemistry, Physical and Theoretical Chemistry Laboratory, University of Oxford, South Parks Road, Oxford OX1 3QZ, United \\ Kingdom \\ ${ }^{2}$ Department of Engineering Science, University of Oxford, Parks Road, Oxford, OX1 3PJ, United Kingdom \\ *Corresponding author: arran.curran@chem.ox.ac.uk
}

Published October 1, 2014

\begin{abstract}
This document provides the supplementary information for 'Decoupled and simultaneous three-dimensional imaging and optical manipulation through a single objective', http://dx.doi. org/10.1364/optica.1.000223. It includes a detailed description of the colloidal particles, the inverted microscope, the holographic optical trapping, the remote refocusing, the confocal scan head and the measurement of the point spread function. (c) 2014 Optical Society of America
\end{abstract}

OCIS codes: $\quad 350.4855$ Optical tweezers or optical manipulation; 180.0180 Microscopy; 180.1790 Confocal microscopy

http://dx.doi.org/10.1364/optica.1.000223.s001

Colloidal particles

The colloidal particles in Fig. $2 b$ and Fig. $3 a$ and $b$ of the Letter are rhodamine B-labeled melamine particles with a diameter of $2.8 \mu \mathrm{m}$ (Microparticles $\mathrm{GmbH}$ ) in demineralised water or ethanol, respectively. For the flow experiments (Fig. 3d-f), we use a system consisting of $10 \mu \mathrm{m}$ diameter polystyrene particles (Microparticles $\mathrm{GmbH}$ ) and $~ 500 \mathrm{~nm}$ diameter rhodamine-B labeled 3-(trimethoxysilyl)propyl methacrylate particles [1] in demineralised water. This system was put into a flow cell made of a $1 \mathrm{~mm}$ square capillary connected to a Harvard microfluidics pump and the tracer particles were tracked using a standard particle tracking algorithm [2].

Inverted microscope

The inverted microscope uses a single oil immersion $60 \times$ objective, O1 (Olympus PlanApo N) with a numerical aperture (NA) of 1.42. Olympus immersion oil is used for all the experiments presented $(n=1.518)$. Two achromatic lenses, $f_{1}=200 \mathrm{~mm}$ and $f_{4}=250 \mathrm{~mm}$ act as the tube lenses for the visible imaging and the near-infrared (NIR) optical trapping, respectively. Two dichroic mirrors direct the visible (Semrock BrightLine) and NIR (Thorlabs DMSP1000) optical paths into the back aperture of O1. For brightfield imaging, Köhler LED illumination is mounted above the sample.
Holographic optical trapping

A Boulder Nonlinear Systems spatial light modulator (SLM) is used to manipulate the wavefront of the trapping laser, emitting at a wavelength of $1064 \mathrm{~nm}$. A personal computer connected to the SLM via a DVI connection, generates a phase map using the addition of various diffraction gratings and lenses to produce multiple diffracted beams, each of which form a separate optical trap [3].

Optical remote refocusing

The optical remote refocusing comprises of two air-immersion $50 \times$ objectives (O2 and O3, Olympus MPLAPON, NA = 0.95) that are mounted nose-to-nose. O2 and O3 are not coverslip corrected. Objective $\mathrm{O} 3$ is mounted on a nano-positioning piezo (PI P-726 PIFOC High-Load Objective Scanner) allowing high precision placement of the objective with a range of $100 \mu \mathrm{m} \pm$ $0.3 \mathrm{~nm}$. The range of our positioning device is demagnified by a factor of 1.5 between $\mathrm{O} 2$ and $\mathrm{O} 1$ giving an axial refocusing distance at the sample of $66.7 \mu \mathrm{m}$. A fundamental limit on the axial depth can be determined from Eq. 23 of Ref. [4] by setting the Strehl ratio $S$ to the standard for acceptable image quality, $S=0.8$. For our system, an axial depth limit of $140 \mu \mathrm{m}$ is obtained. An achromatic lens of focal length $180 \mathrm{~mm}$ (nominal 


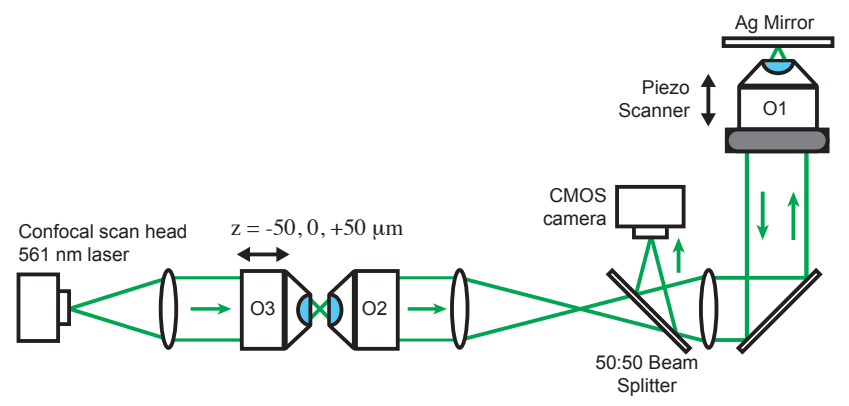

Fig. S1. Setup for determining the point spread function. To measure the point spread function, $\mathrm{O} 1$ is mounted on a piezoscanner and a 50:50 beam splitter is used to reflect the image off the Ag mirror which is placed at the sample plane of O1. Laser light $(561 \mathrm{~nm})$ from the confocal scan head is sent down the optical axis of the microscope and focused onto the Ag mirror. The reflected light, which is imaged onto a CMOS camera, is the in-plane light distribution at the sample.

Olympus tube length) is used as the tube lens for O3. Objectives $\mathrm{O} 1$ and $\mathrm{O} 2$ are imaged onto each other by a $4 f$ system with a magnification given by $M_{4 f}=f_{1} / f_{2}=n_{1} F_{1} M_{2} / n_{2} F_{2} M_{1}=1.25$ [4], where $F_{1,2}=F=180 \mathrm{~mm}$ is the nominal tube lens focal length of the objectives with magnification $M_{1,2}$ and immersion media refractive indices $n_{1,2}$. The focal lengths of the achromatic lenses are $f_{1}=200 \mathrm{~mm}$ and $f_{2}=160 \mathrm{~mm}$. The chief ray from a point emitter laterally displaced by $100 \mu \mathrm{m}$ from the optical axes at the sample plane will exit the back aperture of $\mathrm{O} 1$ at an angle of $1.9^{\circ}$ and is relayed to the back aperture of $\mathrm{O} 2$ at an angle of $2.4^{\circ}$. In other words, a $100 \mu \mathrm{m}$ off-axis point at the image plane of $\mathrm{O} 1$ is imaged at the front focal plane of $\mathrm{O} 2$ with a lateral displacement of $150 \mu \mathrm{m}$ which is well with the maximum field of view of $\mathrm{O} 2$. For our system we have a magnification of $M_{1} f_{1} / F=67 \times$ at the first image plane which is then demagnified by $M_{2} f_{2} / F=44 \times$ resulting in an overall magnification of $1.5 \times M_{3}=75 \times$.

\section{Confocal scan head}

The confocal scan head is a resonant $(8 \mathrm{kHz})$ point scanning system designed and developed by Thorlabs (customised CLS-3HS). The $561 \mathrm{~nm}$ excitation laser reflects off the resonant scanner and galvanometer before leaving the confocal scan head and exciting the sample and the returning fluorescence is then de-scanned through the scanning optics. The fluorescence is then focused through the pin hole of the confocal scan head and coupled into a multi-mode optical fibre where a photomultiplier tube detects the signal. The imaging speed of the confocal scan head is 30 frames per second bidirectional at a resolution of $512 \times 512$ pixels. For the velocity field data presented in Fig. $3 \mathrm{~d}$ uni-directional scanning was used with a field size of 832 $x 832$ pixels and a pixel resolution of $0.08 \mu \mathrm{m}$. This gives an acquisition time of $54 \mathrm{~ms}$ per frame. For the flow velocities we applied, the $500 \mathrm{~nm}$ diameter tracer particles travel a distance of up to their diameter between subsequent frames. Hence, on the time scale of image acquisition the motion of the tracer particles is significantly smaller than the average distance between the particles in the dilute suspension, which allows us to readily determine the velocity fields from our confocal images.
Measuring the point spread function

The performance of our microscope is characterised by determining the point spread function (PSF). This is done by measuring the intensity profile of a focused laser at the sample plane of O1, since the intensity profile equates to the PSF [4, 5]. To measure the intensity profiles we modify the microscope by mounting $\mathrm{O} 1$ on a piezo-scanning device and placing a Ag mirror at the sample plane. Figure S1 shows a schematic of the modified microscope for measuring the PSF. Laser light is sent down the optical axis of the microscope from the confocal scan head. A 50:50 beam splitter placed between O1 and O2 directs laser light that has travelled down the microscope and reflected back by the Ag mirror, onto a CMOS camera. The image formed on the CMOS camera is the in-plane light distribution of the focused laser beam at the sample. O1 is then scanned along the optical axis to give a three-dimensional image of the intensity distribution. The images presented in the manuscript are the axial section of these three-dimensional images for three positions of $\mathrm{O} 3$ as discussed in the manuscript. The images presented are visually confirmed to be free from spherical aberration. For an example of a remote refocusing system with and without spherical aberration see Fig. 8 of reference [4]. Given the visual confirmation that the intensity profile is aberration free we then estimate the diffraction limited spot size (diameter) of $\mathrm{O} 1$ to be $1.22 \times \lambda / N A=1.22 \times 1064$ $\mathrm{nm} / 1.42=914 \mathrm{~nm}$, which compares very well to vertical width of the intensity profiles in Fig. 1c. These two points show that our system is aberration free and diffraction limited.

\section{REFERENCES}

1. S. Sacanna, W. T. M. Irvine, P. M. Chaikin, and D. J. Pine, Nature 464, 575 (2010).

2. J. C. Crocker and D. G. Grier, J. Colloid Interf. Sci. 179, 298 (1996).

3. R. W. Bowman, G. M. Gibson, A. Linnenberger, D. B. Phillips, J. A. Grieve, D. M. Carberry, S. Serati, M. J. Miles, and M. J. Padgett, Comput. Phys. Commun. 185, 268 (2014).

4. E. J. Botcherby, R. Juškaitis, M. J. Booth, and T. Wilson, Opt. Commun. (2008).

5. T. Wilson, Confocal Microscopy (Academic Press, 1990). 